Li Wan

Natalie Yang

Chee-Yan Hiew

Anthony Schelleman

Lynne Johnson

Clive May

Rinaldo Bellomo

\section{Reply to Meco et al.}

Accepted: 28 March 2009

Published online: 7 May 2009

(C) Springer-Verlag 2009

This reply refers to the comment available at: doi:10.1007/s00134-009-1497-1.

We thank Dr. Meco and colleagues for their thoughtful letter.

We apologize for the two typing errors that unfortunately slipped through despite our careful reading of the manuscript, our reviewer's thorough checks and editorial review.
RBF was, of course measured in $\mathrm{ml} / \mathrm{min}$ and the formula used to calculate RBF was, of course, based on the area of the renal artery not the radius (we assume the correspondents mean "radius" when using the word "ray").

Finally, the heart rate (HR) is intrinsic to how the area under the curve (AUC) for RBF is measured by the MEDARCHIVE program used for our calculations: five continuous waveforms are used to calculate the AUC for five waveforms, and the AUC is divided by the time taken (i.e. HR) for those five waveforms to occur, thus the AUC quoted is a calculated average (i.e. HR is factored in) adjusted to an equivalent rate of one beat per second and thus only then multiplied by 60 to calculate blood flow per minute.

Accordingly, we believe that our findings are true [1] and fully justify our conclusions.

\section{Reference}

1. Wan L, Yang N, Hiew C-Y, Schelleman A, Johnson L, May C, Bellomo R (2008) An assessment of the accuracy of renal blood flow estimation by Doppler ultrasound. Intensive Care Med 34:15031510

L. Wan · R. Bellomo (

Department of Intensive Care, Austin Hospital, Heidelberg, VIC 3084, Australia e-mail: rinaldo.bellomo@austin.org.au Tel.: +61-39-4965992

Fax: +61-39-4963932

N. Yang - C.-Y. Hiew · A. Schelleman · L. Johnson

Department of Radiology, Austin Hospital, Heidelberg, VIC, Australia

C. May

Howard Florey Institute,

University of Melbourne, Melbourne,

VIC, Australia 